\section{Science should help teach children the meaning of humanity}

Sir - The scientific community must push forward new curriculum ideas before more US school boards like those in Kansas and Kentucky attempt to deny their students a proper science education ${ }^{1}$. Rapid changes in the world economy and in social structures are prompting the need for individuals who can adjust and thrive in a changing world. These challenges require educational innovation. Yet according to international test scores, US public schools are stagnating.

In 1963, the view emerged that "the rate of change in the society in which we live forces us to redefine how we shall educate a new generation" ${ }^{2}$. Environmental problems, population growth, violence and growing suburbanization were placing increasing demands on resources. These demands prompted the creation of a curriculum designed to use education, rather than government regulation and penalties, to improve the human condition $^{2}$.

The result was Macos (Man: a course of study), a school programme integrating anthropology, biology and the latest concepts of educational psychology. Developed by teachers and specialists in the areas studied, with the help of a grant from the National Science Foundation (NSF), it stayed in use for more than a decade. At its peak the Macos curriculum was taught in 47 states and 1,700 schools, and was hailed as significant educational progress ${ }^{3}$. Forty years later, the same social and economic problems remain. Perhaps it is time for the social and biological sciences again to be made central to a multidisciplinary curriculum based on complex systems of human societies and behaviour.

Macos attempted to achieve social change by the use of anthropological and biological models designed "to help children understand what it means to be human"2 by addressing basic questions about humanity. Five goals were established: to give pupils respect for and confidence in the powers of their own minds; to use this to give them power to think about the human condition and society; to provide workable models to analyse the nature of society and the human condition; to impart respect for the capacities and humanity of the human race as a species; and to provide a sense of the unfinished business of human evolution ${ }^{2}$.

Macos was a daily 40-45-minute lesson for fifth- and sixth-grade students (aged 9 to 11). The course included studying the life and culture of the Netsilik Eskimos and their habitat. By examining the biological and anthropological systems of the Eskimos, children could examine their own culture and habitat ${ }^{4}$. The course was discontinued in 1976 by the NSF, prompted by Senator John Conlan (Republican, Arizona), who objected to it because it dealt with topics such as evolution, reproduction and violence 5 .

In essence, Macos was a beginning course in philosophy, using several disciplines to address basic questions of humanity, focusing on creating complex questions rather than answering them. Fact acquisition was secondary to establishing a foundation on which learning and thinking would grow. While the idea of creating questions instead of delivering facts may be frowned on by proponents of standardized tests, it is essential for teaching children to think.

An educational curriculum needs to be designed today that has the same character and purpose as Macos, but based on broader, complex systems, with computer databases and the Internet used as integral tools by teachers. The development of the curriculum must be a collaborative effort by leaders of several disciplines to provide a comprehensive and successful educational programme . $^{6}$.

Scientists must not sit back and watch movements towards irrationality, such as the judgements in Kansas and Kentucky in 1999. A united scientific community can provide an impetus for future social change by introducing and supporting a strong science curriculum based on complex systems.

\section{Greg Kontos}

Information Technology Department, College of William and Mary, Jones Hall, Room 105,

Williamsburg, Virginia 23185, USA

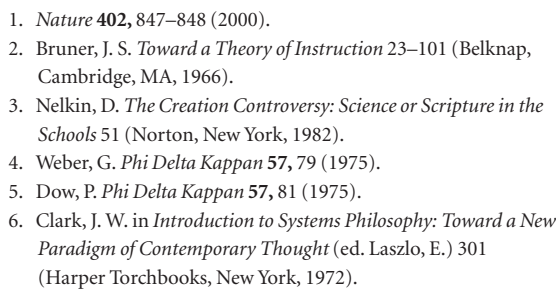

\section{Particle physicists need a common objective}

Sir - I would like to comment on two points arising from your Opinion article and News story on the TESLA project (Nature 410; 395 \& 397; 2001).

First, the cost of 3,136 million euros (US $\$ 2,811$ million) for the TESLA 500-GeV electron-positron linear collider is based on experience of construction of a complete test facility, on operation for four years, and on a full costing performed by industry, including the effects of mass production.

I disagree with your opinion that the cost will double when labour and operation costs are included, because all components will be fabricated by industry and the associated labour costs are included in the price. Testing and assembly of components will require roughly 7,000 person-years, the cost of which will have to be added to the investment cost, and which amounts to no more than $10-20 \%$ of the total cost, depending on national salary scales. Operating costs are not included in any of the existing cost estimates: for TESLA these should be 120 million euros a year, or $3 \%$ of the investment.

Second, you state in your editorial on the German, US and Japanese plans for linear accelerators that Europeans should accept that they have had their turn with CERN's Large Hadron Collider. Your News story says that TESLA in Germany "could mark the virtual eclipse of particle physics in the United States and Japan for a generation". In my view, regional balance is good but not vital for a field. This has been demonstrated successfully by astronomers who go where the sky tells them, without endangering national programmes.

In particle physics we need a new model of international collaboration to be able to build and operate accelerators as global endeavours, in which all partners have shares and a say, thus making a facility for all countries, independent of the location. It is important that particle physicists everywhere get behind one common project, and convince their governments to fund and build it wherever the strongest political support is found.

Albrecht Wagner

DESY, D-22603 Hamburg, Germany

\section{No campaign to strip Baltimore of his Nobel}

Sir - In his review of Ahead of the Curve, Shane Crotty's biography of David Baltimore (Nature 410, 746; 2001), Robert Bazell perpetuates a misunderstanding of my role in the 'Baltimore case' when he writes that I "campaigned to have Baltimore's Nobel prize rescinded and to have him expelled from the National Academy of Sciences". The assertion that I led a campaign to destroy Baltimore is false. Its origin may lie in the reappearance at that time of the long-discredited rumour that David knew of Howard Temin's discovery of avian reverse transcriptase before he isolated its murine equivalent - I had inappropriately repeated what turned out to be an unfounded story. 
Earlier, before the Dingell congressional hearings under which David was investigated for his support of a colleague accused of scientific fraud, I had in fact tried to defuse the situation, first by holding a small meeting on scientific integrity at our Banbury Center to try to review what was going on. Although invited, David declined to come, but his close associates did. Second, I went to the offices of Congressman Norman Lent, Long Island's Republican counterpart to John Dingell, to tell him that the controversy involved not fraud but possible mistakes of scientific judgment, and as such it should not merit the attention of a congressional committee.

I have only the highest respect for Baltimore as a scientist and leader of science, but his intransigence at the time made many of us worry that the affair was harming not only himself but US science as well. I in no way feel apologetic for not supporting behaviour that I feel is antithetical to the scientific tradition in which I was raised. The Harvard professors who saw the need to publicly question David's behaviour only reluctantly joined the fray. In reporting their and my behaviour as "horrid", Bazell does us a great injustice. Under no circumstances could John Edsall or Paul Doty, say, be accused of jealousy of a peer's meteoric career. I and the Harvard professors were deeply bothered by what seemed to us actions not appropriate for an individual of such talents. I totally concurred in my former colleagues' forthright wish for justice to prevail.

James D. Watson

Cold Spring Harbor Laboratory, PO Box 100, Cold Spring Harbor, New York 11724, USA

\section{How inbreeding affects productivity in Europe}

Sir - Navarro and Rivero in Correspondence ${ }^{1}$ for the first time quantified favouritism ("inbreeding") in Spanish universities, showing that it is at least 10 times higher than in France, the United States and the United Kingdom. I have now quantified this phenomenon in 51 universities from 14 European countries.

I examined the relationship between the percentage of papers published by each country and its level of inbreeding. I gathered information from 51 ecology or zoology departments via a brief questionnaire requesting information on the number and rank of teaching positions, as well as the number of these staff trained at the same university.
I obtained responses from at least two universities in each country. I estimated "inbreeding" as the percentage of staff in these positions trained at the university. I found significantly more variance in inbreeding among than within countries $(F=12.38$, d.f. $=13,37, P<0.0000001$; one-way analysis of variance), showing that countries are consistent in their degree of favouritism.

Spain registered the second highest level of inbreeding, averaging $88 \%$, surpassed only by Portugal (91\%). Figures for the remainder are (in \%): Italy (78), Austria (73), France (65), Norway (56), Belgium (52), Finland (48), Netherlands (40), Denmark (39), Sweden (32), Switzerland (23), United Kingdom (5.2) and Germany (1).

To explore the relationship between level of inbreeding and scientific productivity, I used May's enlightening report ${ }^{2}$, in which he assesses the quality of the contribution of various countries to world scientific knowledge. May provides information about the share of papers and citations in science, medicine and engineering provided by 15 countries, not including Portugal, Spain, Austria, Norway and Belgium. If, conservatively, I assign these countries a value of 0.5 , a significantly negative correlation appears between the percentage of papers published by each country and its level of inbreeding $\left(R_{\mathrm{s}}=-0.60, P=0.02, n=14\right)$. That is, overall scientific productivity correlates negatively with the percentage of inbreeding.

In the 1980s the Spanish government attempted to end inbreeding in its universities by the University Reform Law (LRU). The Real Decreto 1888/1984 of 26 September, which regulates the employment system in Spanish universities, clearly states: "The research activities of the candidates shall be evaluated as the priority merit" (Art. 8.2.a). This reform has, however, failed to change the system ${ }^{3}$; indeed, the problem has become even more serious, as pointed out in two Nature editorials ${ }^{4,5}$.

The inbreeding system is extremely stable. The solution needs vigorous measures: first, every position should be advertised internationally; second, there should be no local members on appointment committees; and third, lecturers or full professors with low scientific productivity should not serve on committees that appoint professorships. As things stand, Spanish universities are autonomous and they do not want the system to change because, in general, university politicians (people who manage universities) are not good researchers and do not consider that scientific productivity is paramount.

When will the Spanish government decide to implement the necessary drastic reforms?

Manuel Soler

Department of Animal and Ecological Biology,

Faculty of Sciences, University of Granada, 18001, Granada, Spain

1. Navarro, A. \& Rivero, A. Nature 410, 14 (2001).

2. May, R. M. Science 275, 793-796 (1997).

3. Rodilla, V. Nature 376, 290 (1997)

4. Nature 389, 767 (1997).

5. Nature 396, 709 (1998).

\section{Philanthropists are paying their dues}

Sir - I would like to add some points to your excellent News Feature on biomedical philanthropy (Nature 410, 140-143; 2001), which highlighted the fact that high-risk research not funded by the National Institutes of Health (NIH) is increasingly being supported by philanthropists.

First, the NIH system is one of the best biomedical research funding systems in the world. NIH support of research and research training has catalysed much of the explosion of biomedical knowledge over the past few decades.

Second, the NIH and other US agencies have freely allowed investigators to patent technological discoveries made while they were supported by government funds, a policy responsible for the great success of many entrepreneurs. It is thus only fair that some of their good fortune is funnelled back to support basic research.

Third, over the past few years the NIH has been seeking and supporting high-risk research, for example the National Eye Institute's new R03 programme, the National Institute on Drug Abuse's CEBRA programme and the R21 programme of the National Institute of Mental Health and National Institute of Neurological Disorders and Stroke.

Programme officers from each of these institutes have personally contacted me to inform me of these initiatives and to encourage me to apply. My own lab's high-risk work has received funding from both NIH and philanthropic sources.

Finally, and perhaps most important, the NIH system is only as good as the quality of peer review. If biomedical researchers are unhappy with the quality of the review process, we need only look to ourselves to improve it.

Ben A. Barres

Department of Neurobiology, Stanford University School of Medicine, Fairchild D235, 299 Campus

Drive, Stanford, California 94305-5125, USA 